\title{
THE ELECTRICAL STIMULATION OF MOTOR NERVE AND MUSCLE
}

SomE of the effects of electricity upon living tissue have been noted since ancient times, but only since the time of Galvani and Faraday have such effects begun to be understood as knowledge of physics and physiology has grown. Many ambitious claims were made in the eighteenth and the nineteenth centuries for the therapeutic effects of electricity but the direct use of stimulation to produce function has depended on advances in electrophysiology and micro-electronics during the present century. While pacemakers for cardiac muscle are now well established, the control of smooth and skeletal muscle poses a far more difficult problem and has only recently become possible through progress in computing, robotics and a better understanding of muscle properties.

In the absence of a full understanding of such properties one of the most elusive goals has been the production of predictable levels of force by given patterns of stimulation. Muscle can be made to contract by stimuli delivered from electrodes applied to the overlying skin, the motor nerve or the muscle itself, in each case the threshold for response being much lower for the motor neurons than for the muscle fibres. It is therefore desirable that patients should have intact lower motor neurons to the relevant muscles. Electrodes applied to nerve or muscle may be linked by transcutaneous wires to stimulators outside the body or connected to implanted stimulators controlled and powered by external radio transmitters.

The potential of such technology has generated an understandable interest in the general public particularly in relation to patients with spinal cord injury who may well be suitable subjects because their lesions are generally non-progressive and involve mainly upper motor neurons. Stimulation may be used to enhance contraction in patients with incomplete lesions or in an attempt to control it completely below the level of a complete lesion.

The stimulation of the phrenic nerve to maintain diaphragmatic respiration in patients with cord lesions above $\mathrm{C}_{4}$ has a place in patients requiring long-term ventilatory support if the diaphragm responds well to trial stimulation and the patient's cerebral function is normal or near normal (Glenn et al., I980). After trials with surface stimulation, individual cuffs are placed on each phrenic nerve and stimulated by a radio-controlled implant. The two sides may be stimulated individually or together and technically the problem is a relatively simple one of on/off control. Intermittent positive pressure ventilation retains certain advantages however of more uniform expansion and more direct measurement of respiratory parameters.

The artificial control of the urinary bladder and sphincters is of great potential significance to a minority of spinal cord injury patients in whom existing methods have failed to permit micturition, continence or the avoidance of chronic infection. As with the diaphragm this is to some extent a problem of on/off control but micturition entails complex reflexes and it may be difficult to separate the responses of bladder and sphincters. 
Following experimentation with stimulation the bladder wall, the conus medullaris and the sacral nerves, considerable reliability has now been achieved particularly in stimulating the anterior roots of the sacral nerves using a radio controlled implant for voiding and sometimes for continence, with additional beneficial effects in some patients on erection and bowel habit. (Brindley et al., I982).

In the case of skeletal muscle, much more accurate proportional control of force is required for useful function and the effects of fatigue, temperature, and muscle length combine to render the theoretical basis of such control formidably complex. In addition the co-ordination of multiple muscles may be required in a dynamically varying pattern of position and load. Digital computers are virtually essential for the solution of such problems. In general the patterns required by a particular patient will need to be developed on a laboratory computer and then transferred to a portable computer transmitting them to an implanted stimulator. The prospect of control of the lower limb has roused interest in the possibility of walking, although this entails formidable complexities of balance, proprioception and energy expenditure. It may, however, be reasonable within the next few years to expect practical benefit from the ability to stand up and to take a few steps, although a walking frame or crutches are likely to be needed. (Marsolais and Kobetic, I983).

Simple limb control may be more necessary and more beneficial in the case of tetraplegic patients if simple grasp and release functions can be restored to the hand (Peckham et al., I980). The small muscle groups involved will require implanted electrodes under radio control and such technology will be available in the next few years. Skin surface electrodes may well be adequate for simple leg movements and for exercise and retraining of wasted muscle and much work remains to be done on the physiological and metabolic effects of such exercise.

There remain considerable problems of replacing not only motor nerve function but the sensations of touch and proprioception. Special sensors require to be developed and their output fed back to modify patterns of stimultion via potentially complex control equations. In tetraplegics in particular there is a shortage of sites which can be used for the voluntary initiation of movement. Nevertheless there is hope for the restoration of simple functions during the next decade. In the meantime, it behoves all those involved in the care of spinal injury patients to prevent contractures, preserve lower motor neurons and muscle where possible, and perhaps to strengthen wasted muscle and osteoporotic bone through structured programmes of electrically stimulated exercise.

\section{REFERENCES}

Brindley, G. S., Polkey, C. E. \& Rushton, D. N. (I982). Sacral anterior root stimulators for bladder control in paraplegia. Paraplegia, 20, 365-38 I.

GlenN, W. W. L., Hogan, J. F. \& Phelps, M. L. (I980). Ventilatory support of the quadriplegic patient with respiratory paralysis by diaphragm pacing. Surgical Clinics of North America, Vol. 6o, No. 5, I055-1078.

Marsolais, E. B. \& Kobetic, R. (I983). Functional walking in paralysed patients by means of electrical stimulation. Clinical Orthopaedics, 175, 30-36.

Peckham, P. H., Marsolais, E. B. \& Mortimer, J. T. (I980). Restoration of key grip and release in the C6 tetraplegic patient through functional electrical stimulation. $\mathcal{F}$. Hand Surgery, Vol. 5, No. 5, 462-469.

Graham Creasey, F.R.C.S.E.

Spinal Unit, Edenhall Hospital, Edinburgh. 